\title{
On variation of the latitudinal structure of the solar convection zone
}

\author{
H. M. Antia ${ }^{1}$, S. M. Chitre ${ }^{2}$, and M. J. Thompson ${ }^{3}$ \\ 1 Tata Institute of Fundamental Research, Homi Bhabha Road, Mumbai 400005, India \\ 2 Department of Physics, University of Mumbai, Mumbai 400098, India \\ 3 Space and Atmospheric Physics Group, The Blackett Laboratory, Imperial College, London SW7 2BW, UK
}

Received 26 September 2002 / Accepted 22 November 2002

\begin{abstract}
The latitudinal sound-speed structure of the Sun's convection zone gives insight into the physical processes occurring there, specifically the cellular convection and possibly the presence of magnetic fields. Using helioseismic data from the GONG network and MDI instrument on SOHO, we map the latitudinal acoustic structure of the convection zone from 1995 to 2002. The temporally averaged structure confirms previous findings of an excess in sound speed at the $10^{-4}$ level at 60 degrees latitude. There also appear to be some variation with time, with the peak in sound-speed asphericity at 60 degrees growing towards the maximum of solar activity according to the MDI data. However, we present some evidence that such variation may be associated with instrumental variation between the epochs before and after SOHO was temporarily lost in 1998. Nonetheless, some genuine temporal variation may be present, and we discuss the possible physical causes of that.
\end{abstract}

Key words. Sun: oscillations - Sun: interior - Sun: rotation - Sun: activity - methods: data analysis

\section{Introduction}

Analysis of the global $p$-mode oscillations of the Sun reveals the Sun's internal structure and constrains the physics of the solar interior. In this paper we focus on the latitudinal variation of the structure of the solar convection zone and the possible temporal variation of that structure during the rising phase of solar cycle 23 . The convection zone occupies the outer thirty per cent by radius of the Sun, as was deduced from helioseismic inversions for the Sun's radial hydrostatic structure (Christensen-Dalsgaard et al. 1991; cf., Gough 1977; Ulrich $\&$ Rhodes 1977). Such an investigation uses the mean multiplet $p$-mode frequencies to deduce the spherically symmetric component of the Sun's structure. But with high-quality data such as from the Global Oscillation Network Group (GONG) and the Michelson Doppler Imager (MDI) instrument on board the SOHO satellite (Harvey et al. 1996; Scherrer et al. 1995) it has become possible to make deductions also about the latitudinal variation of the structure from the dependence on the azimuthal order of the frequencies within multiplets (Gough et al. 1996; Antia et al. 2001). What is measured is acoustic asphericity, i.e., the deviation from spherical symmetry of the propagation speed of the $p$-mode waves as a function of position. The relevant wave speed is the adiabatic sound speed but possibly modified by magnetic effects in regions of magnetic fields. The acoustic structure of the convection zone may be expected to

Send offprint requests to: $\mathrm{H}$. M. Antia, e-mail: antia@tifr.res.in reflect the asphericity caused by the Sun's rotation, but also perhaps the cellular convection and the presence of magnetic fields. At the resolution scale at which the global helioseismic inversions can be undertaken, we are not here concerned with granular or even supergranular scales. But the thermal effects of giant cells or other such large-scale circulatory structures may be detectable in the inferred latitudinal variation of sound speed, as perhaps may also be a sufficiently strong large-scale magnetic field. In the present paper we present results of inversions of a more extensive data set than was considered by Antia et al. (2001). We also show that there is some evidence for the temporal variation of the latitudinal structure during the rising phase of the solar activity cycle. However, one should be cautious because of possible instrumental variation during the period of observations, and so we wish to examine such a possibility more closely.

\section{Seismic data and inversions}

The helioseismic datasets comprise measures of frequencies (so-called even a-coefficients) from GONG and MDI. The modes are identified by radial order $n$, spherical harmonic degree $l$ and azimuthal order $m$. The frequencies $v_{n l m}$ are grouped in multiplets, corresponding to modes with all azimuthal orders (i.e., $m=0, \pm 1, \pm 2, \ldots, \pm l$ ) for given $n$ and $l$. In a spherically symmetric star, all frequencies in the multiplet would be equal, but rotation and asphericity raise this degeneracy. Rather than report individual frequencies for every mode in the multiplet, 

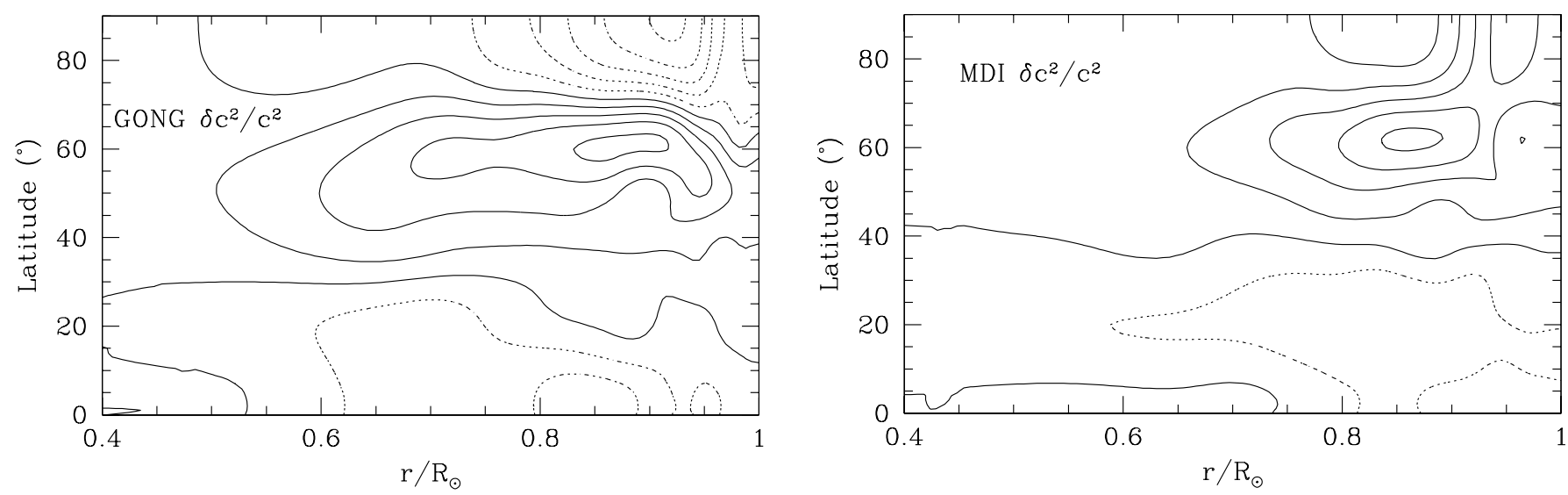

Fig. 1. Contour diagram of the temporal average of aspherical component of squared sound speed as a function of radial distance and latitude using the GONG (left-panel) and MDI (right-panel) data. The MDI data used are restricted to $\ell<110$ (see Sect. 4). Solid and dotted contours denote positive and negative values respectively. Contours are drawn at interval of $2 \times 10^{-5}$.

the observers' data analysis procedure provides mean frequencies $v_{n l}$ and splitting coefficients $a_{j}(n, l)$ defined by

$v_{n l m}=v_{n l}+\sum_{j=1}^{j_{\max }} a_{j}(n, l) \mathcal{P}_{j}^{(l)}(m)$,

where the $\mathcal{P}_{j}^{(l)}(m)$ are orthogonal polynomials in $m$ which are approximately equal to $l$ times Legendre polynomial $P_{j}(m / l)$ when $l$ is large (e.g., Schou et al. 1994).

Odd splitting coefficients $a_{1}, a_{3}$, etc. are used to infer the Sun's internal rotation (e.g., Schou et al. 1998): we shall not consider those further here. The even coefficients contain information about the Sun's aspherical structure, and here we use these coefficients, applying to them a 2D regularised leastsquares inversion technique as described by Antia et al. (2001). In brief, the data are fitted with an expression of the form

$$
\begin{aligned}
\frac{l a_{2 k}(n, l)}{v_{n l}}= & \left(2 k+\frac{1}{2}\right) Q_{l k} \int_{0}^{R} \mathrm{~d} r \int_{0}^{\pi} \sin \theta \mathrm{d} \theta\left(K_{c^{2}, \rho}^{n l}(r) \frac{\delta c^{2}}{c^{2}}(r, \theta)\right. \\
& \left.+K_{\rho, c^{2}}^{n l}(r) \frac{\delta \rho}{\rho}(r, \theta)\right) P_{2 k}(\cos \theta)+Q_{l k} \frac{F_{k}\left(v_{n l}\right)}{E_{n l}} .
\end{aligned}
$$

Here the known quantities are the kernels $K_{c^{2}, \rho}^{n l}$ and $K_{\rho, c^{2}}^{n l}$, the factors $Q_{l k}$, and the mode inertias $E_{n l}$; and the unknowns are the aspherical perturbations $\delta c^{2}$ and $\delta \rho$ to the squared sound speed and density respectively, and the surface terms $F_{k}$. The errors on aspherical perturbations, $\delta c^{2} / c^{2}$ and $\delta \rho / \rho$ are estimated through a Monte-Carlo simulation. The inversion procedure is repeated for different realisations of data, with Gaussian noise added according to the estimated errors on splitting coefficients, to estimate the standard deviation in the values of $\delta c^{2} / c^{2}$ and $\delta \rho / \rho$ at each point.

The solar oscillations frequencies from the GONG project (Hill et al. 1996) were obtained from 108-day time series, while the MDI data sets were derived from 72-day time series (Schou 1999). We use data sets that consist of mean frequencies and splitting coefficients. In this work we are only concerned with even splitting coefficients which are determined by the second order effect of rotation, asphericity in solar structure or of a magnetic field. We use 66 data sets from GONG each covering a period of 108 days, starting from 1995, May 7 and ending on 2002, January 17 with a spacing of 36 days between consecutive data sets. Thus each set overlaps with the two preceding and two following sets. The MDI data sets (Schou 1999) consist of 28 non-overlapping sets each obtained from observations taken over a period of 72 days. The first set begins on 1996 May 1 and the last set ends on 2002 March 30 with a gap in between during July 1998 to January 1999 when contact with SOHO was temporarily lost.

Since the dominant north-south symmetric component of solar rotation rate is reliably determined from odd splitting coefficients, we use these values to calculate the second order effect of rotation as described by Antia et al. (2000). This second order contribution to even splitting coefficients is subtracted from the observed coefficients to get the residuals which could be due to the effect of magnetic field or any other asphericity in solar structure. In all results described in the following sections the rotational contribution has been subtracted out. All these results have been obtained through inversion of even coefficients $a_{2}, a_{4}, \ldots, a_{16}$. The mean frequencies $v_{n l}$ are determined by the spherically symmetric structure. Since this component has been extensively studied we do not consider it in the present work. The even splitting coefficients are sensitive only to the north-south symmetric component of the structure variations and hence that is the only component that can be determined with these data.

\section{Sun's latitudinal structure}

Several investigations of the medium-degree even splitting coefficients in the last several years have pointed to a latitudinally dependent spatial variation in sound propagation speed for the solar $\mathrm{p}$ modes at a depth of about $40 \mathrm{Mm}$ beneath the surface (Antia et al. 2000; Dziembowski et al. 2000). The magnitude of the effect is such that it could correspond to a relative thermal perturbation at that depth of order $10^{-4}$, or a magnetic field with rms field strength of roughly $50 \mathrm{kG}$. Antia et al. (2000) speculate that such a field may be concentrated and retained at that location by downward convective plumes (cf., Li \& Sofia 2001). 


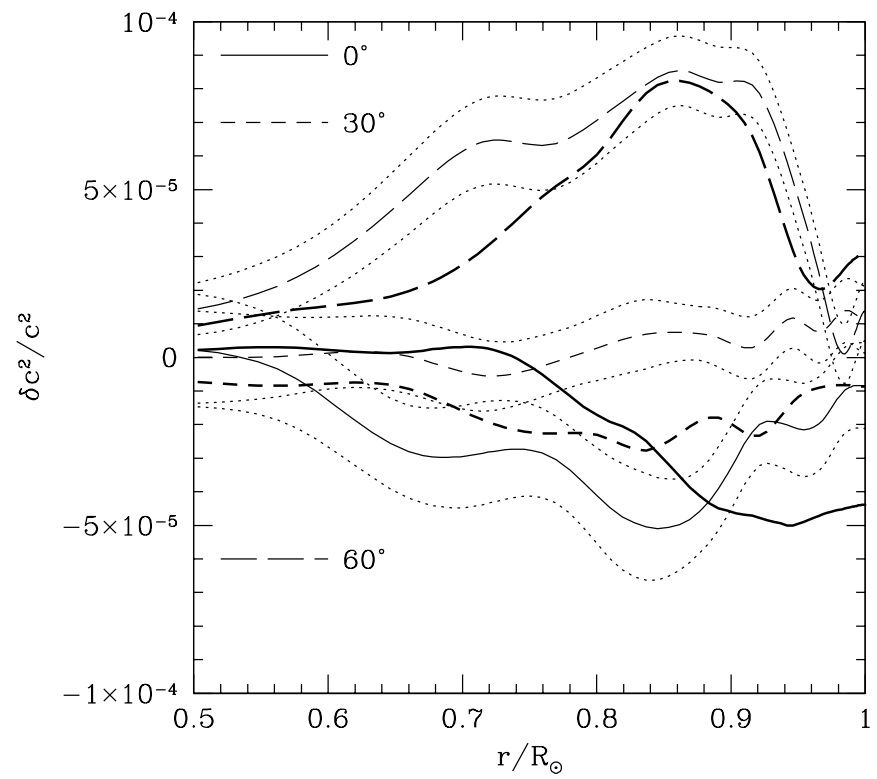

Fig. 2. The aspherical component of squared sound speed as a function of radius at a few selected latitudes as marked in the figure. The thin lines are results using the GONG data with dotted lines showing the error estimates, while the heavy lines are the MDI results. For clarity the errors in MDI data are not shown, but they are comparable to those in GONG data.

The results of 2-D inversion by Antia et al. (2001) strikingly show that the aspherical perturbation to the sound speed peaks at latitude of about $60^{\circ}$, with a drop towards the pole. These results have been updated using additional data that have become available since then: Fig. 1 shows the contours for the temporal average of the aspherical sound-speed perturbation from the updated MDI and GONG data. The MDI and GONG results are in reasonable agreement about the location of the perturbation and its global behaviour. However, note that the period over which the average is taken differs in the two panels because the GONG data start a little earlier (in mid-1995) and there are also two periods without data from MDI.

Figure 2 shows the cuts through Fig. 1 at constant latitudes from both GONG and MDI data. It is clear that the two results are roughly consistent with each other. The cut through $60^{\circ}$ latitude shows a clear peak around $r=0.9 R_{\odot}$. We cannot tell from the seismic data whether this is due to a thermal asphericity in the solar interior or to a magnetic field. If it is due to a randomised magnetic field in the convection zone, then this value may be taken as indicative of the order of magnitude of the ratio $v_{\mathrm{A}}^{2} / c^{2}$, where $v_{\mathrm{A}}$ is the Alfvén speed. From this we can calculate the magnetic field: the results are shown in Fig. 3. Because of the increasing gas pressure the estimated magnetic field keeps increasing with depth. Around the base of the convection zone the magnetic field strength could be 200 $300 \mathrm{kG}$, which is comparable to the upper limit obtained by Antia et al. (2000). The upper limit of Antia et al. is applicable only to a field concentrated near the base of the convection zone, while the magnetic field shown in Fig. 3 is spread throughout the convection zone and hence the earlier upper limit is not applicable.

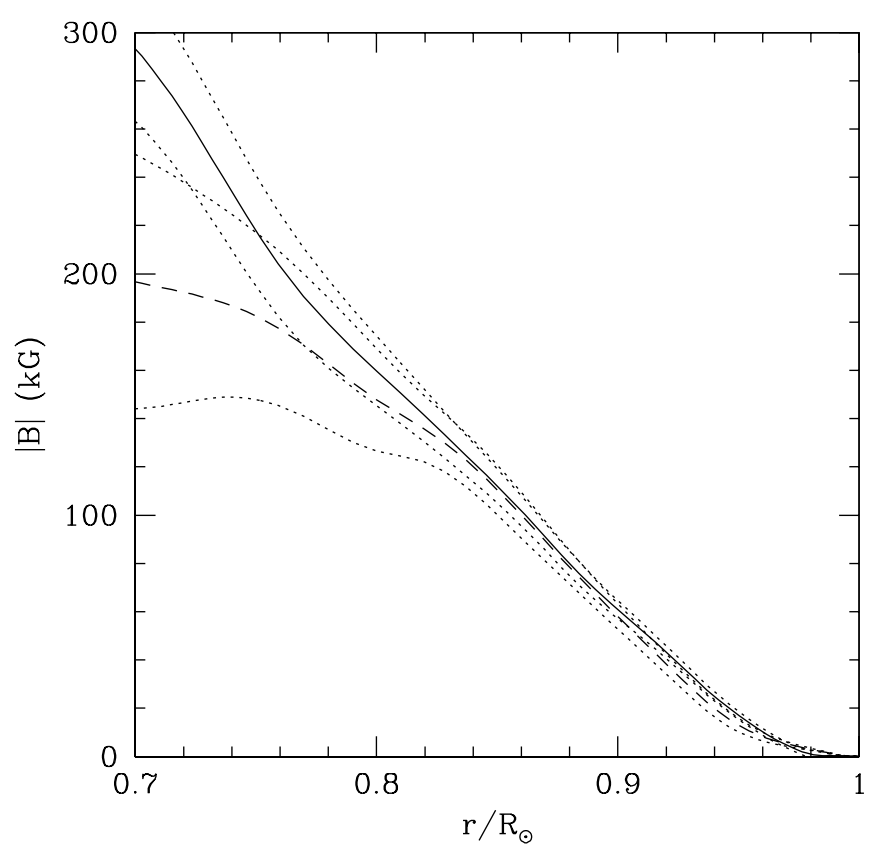

Fig. 3. The estimated magnetic field at $60^{\circ}$ latitude assuming that the inferred asphericity is due to magnetic field. The continuous line shows the results from GONG data, while dashed line shows the results using MDI data. In both cases the error limits are shown by dotted lines.

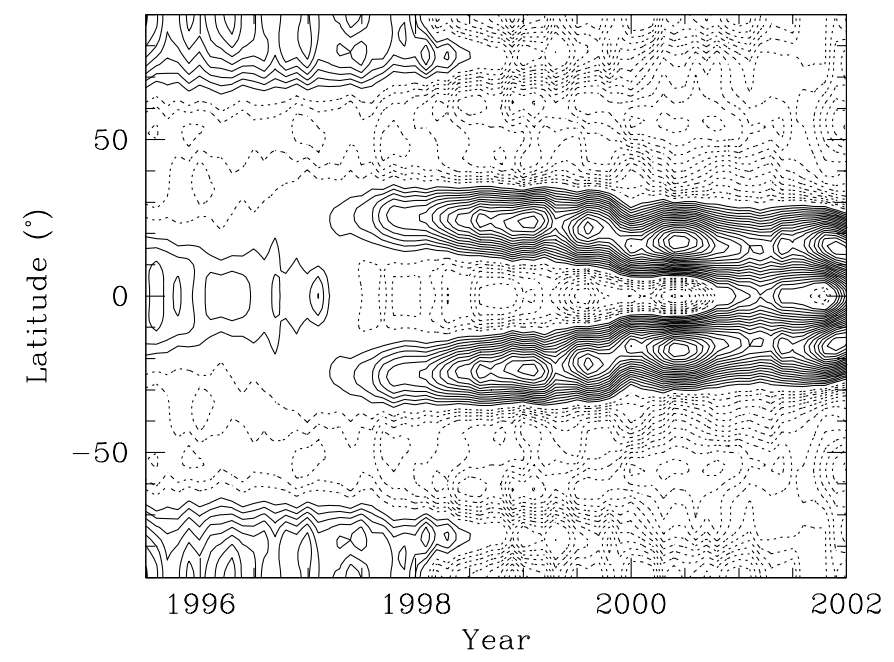

Fig. 4. Contour diagram of the surface term in asphericity inversions as a function of time and latitude using the GONG data. The solid lines show positive contours and dotted lines show negative contours.

The inversion results indicate that the positive perturbation, though peaked around $r=0.9 R_{\odot}$, extends through much of the convection zone. Although the finite resolution of the inversion certainly spreads the features around, it appears from a resolution study (Antia et al. 2001) that the spread in depth seen here is more extensive than one would expect from a perturbation wholly confined around $r=0.95 R_{\odot}$ say. The results appear to show that this peak extends below the convection zone also, but this extension could be due to limited resolution of the inversion process. It may be pointed out that the peak of this perturbation has shifted downwards as compared to what 


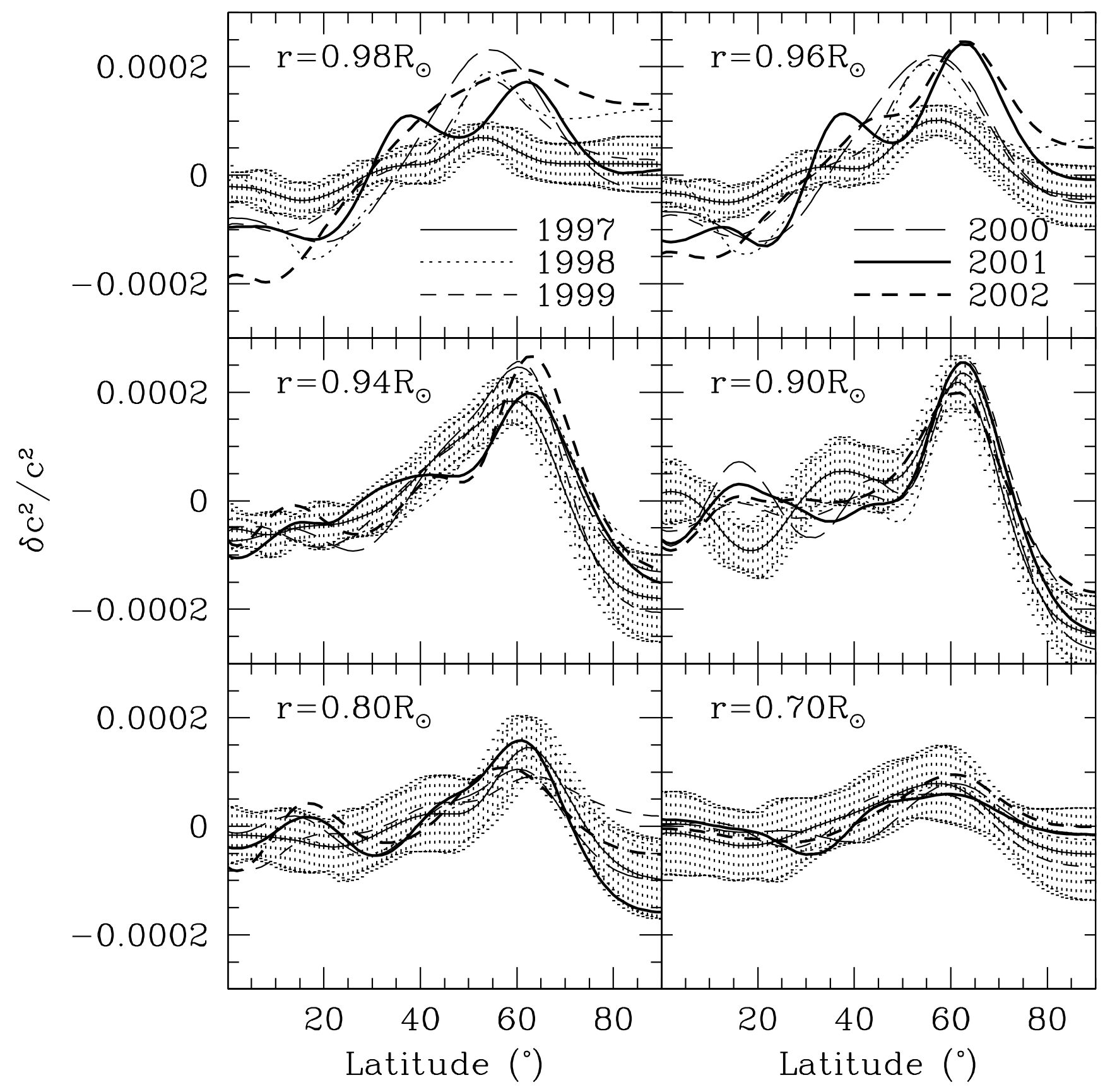

Fig. 5. Latitudinal cuts at fixed radius through the asphericity $\delta c^{2} / c^{2}$ inferred from MDI data, for different radii as indicated in each panel. The $1 \sigma$ error bars are shown for one epoch only as they are similar for all epochs.

Antia et al. (2000) found. This shift has arisen because, unlike in our earlier work, in this work we have removed the surface contribution $\left(Q_{l k} F_{k}\left(v_{n l}\right) / E_{n l}\right)$ during inversion. That shifts the peak a little downwards. If this peak is due to magnetic field, the field strength should be around $70 \mathrm{kG}$.

These results come from an inversion which attempts to isolate the different contributions to Eq. (1). There is a known excellent correlation between the even splitting coefficients $a_{2 k}$ and the surface unsigned magnetic flux projected onto $P_{2 k}(\cos \theta)$. The latitudinal distribution of surface flux varies over the solar cycle. This is reflected in the surface term that we fit in Eq. (1). The surface term is shown in Fig. 4, which displays contours of constant surface term as a function of time and latitude at a frequency of $3 \mathrm{mHz}$. Note that scale and hence the contour spacing of the surface term is arbitrary. This figure shows distinct bands of positive surface term moving towards the equator with time, which should coincide with the bands in the magnetic butterfly diagram. At high latitudes there is a hint of bands migrating polewards, particularly, after 1998. This also has been seen in magnetic features at the solar surface (Leroy \& Noens 1983; Makarov \& Sivaraman 1989b).

\section{Apparent temporal variations}

Clearly there is a substantial temporal variation in the even $a$ coefficients that is associated with the near-surface variations 


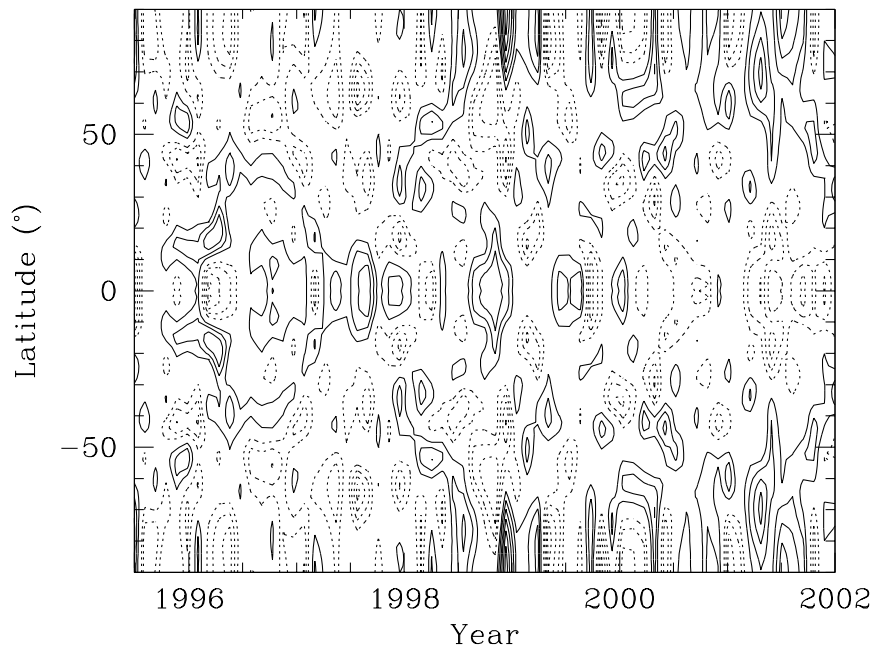

Fig. 6. Contour diagram of the residual in aspherical component of the squared sound speed after subtracting the temporal average, as a function of time and latitude at $r=0.96 R_{\odot}$ using the GONG data. The solid lines show positive contours and dotted lines show negative contours. Contours are drawn at interval of $2 \times 10^{-5}$.

of magnetic flux. This is taken up by the surface term in Eq. (1); see Fig. 4. Once this is removed, however, it is of interest to see whether there is any residual temporal variation coming from the deeper layers.

We first study the development of acoustic asphericity by examining the cuts at different radii from the MDI asphericity inversion results, at six different epochs (the beginnings of 1997, 1998, 1999, 2000, 2001, 2002). Each of these results is obtained from MDI data set starting in the beginning of the corresponding year and represents an average over 72 day period. Figure 5 shows latitudinal cuts at fixed radii $(r / R=0.98,0.96,0.94,0.90,0.80,0.70)$. For the two highest locations, the asphericity clearly appears to evolve, with a pronounced variance at high latitudes. In the $60^{\circ}$ peak, there is a substantial difference between 1997 and later years, and the peak strengthens and broadens approaching 2000. The slightly deeper cuts show the peak sharpening but the polar values are very steady. Much deeper in the convection zone (bottom two panels) the amplitude is much reduced, but it is still somewhat larger than one might expect if the underlying perturbation were strictly confined in the layers around e.g. $0.95 R_{\odot}$. Thus its spread to greater depths may be to some extent real. It is difficult to make out any systematic temporal variation in asphericity from this figure as even in the upper layers the variation is only of the order of $2-3 \sigma$.

Because there is an apparent temporal variation we should look at its origin more closely. Figure 6 shows as a contour plot the results for aspherical component of squared sound speed obtained using GONG data at $r=0.96 R_{\odot}$ as a function of latitude and time. To see the temporal variations more clearly, the figure shows the residuals obtained after subtracting the temporal average at each latitude. There is no evident pattern in the residuals. Similar results are obtained at other depths.

Figure 7 similarly shows the residuals in sound speed at $r=0.96 R_{\odot}$, but using the MDI data. The left panel which displays the results using full data shows a distinct temporal variation. This is consistent with what was seen in Fig. 5, but is in contrast to the GONG results (Fig. 6). Antia (2002) has pointed out that most of the temporal variation occurs between July 1998 and January 1999, when the contact with SOHO satellite was lost. This period is marked by the gap in the contour diagram. Before the gap there is positive asphericity at low latitudes and negative values at high latitudes. This is reversed after the gap and in fact there is very little systematic variations in the results before or after the gap. It is possible the Sun has had some interesting transition while the MDI instrument was not operating. This hypothesis gets little support from the GONG data, however, which does not appear to show any unusual trend during this period. We must therefore consider the possibility that the apparent variation is due to systematic errors introduced by instrumental variations during the recovery of the SOHO satellite. We suspect the high-degree data may be responsible: the right panel of Fig. 7, which shows results using only modes with $\ell<110$, displays no particular temporal variation in asphericity. In fact, the temporal average for the MDI data shown in Fig. 1 is also obtained using only modes with $\ell<110$. If all modes are used then the peak is much more pronounced with maximum value of more than $2 \times 10^{-4}$ which is double the peak height in Fig. 1. As a result, in the following results we have used only the restricted mode set from MDI.

With a view to studying the possibility of small temporal variation in inferred asphericity in sound speed, we divide the data sets into two parts: a low activity period before 1998.4 and a high activity period after 1999.4, and take temporal averages separately over these parts. The results are shown in Fig. 8. There is a slight increase in asphericity in high activity set as compared to that in low activity set in both GONG and MDI results. Figure 8 also shows the difference between the high and low activity periods. The increase appears at a level of $\sim 2 \times 10^{-5}$ in some regions and is spread over a wide region, though the maximum change appears to occur around $r=0.9 R_{\odot}$ at high latitude as well as in the equatorial region. By comparison, the typical errors in low latitude region inside the convection zone is about $10^{-5}$ which is half the contour spacing. The errors increase with depth and latitude. The significance of this increase in asphericity is not very clear, but with the availability of data over a complete solar cycle it may be possible to confirm whether there is indeed an increase in asphericity with activity.

\section{Discussion}

The helioseismic inversions for asphericity in sound speed show a distinct peak at a latitude of $60^{\circ}$ inside the convection zone. These inferences raise a number of questions. What is so special about $60^{\circ}$ latitude? Perhaps it is the boundary between two oppositely directed meridionally circulating cells, or at least the poleward limit of a low-latitude cell. If the perturbation to the sound speed is indeed caused by magnetic field, as we have suggested (Antia et al. 2000), then the flux could accumulate at this latitude in a convergent lane between the two cells. We can envisage two possibilities. Earlier studies of surface magnetism emphasised the poleward migration of prominences and faculae from around this location (e.g., 

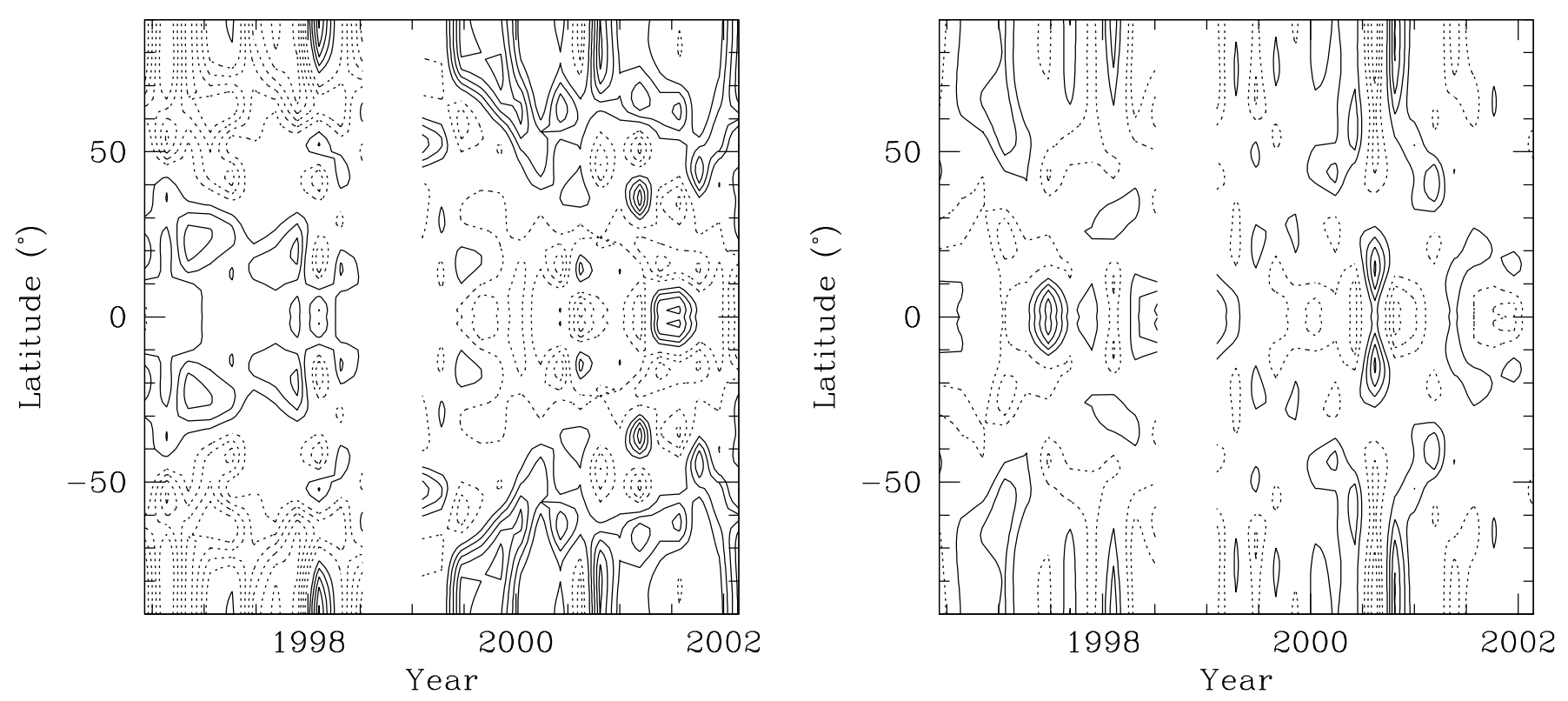

Fig. 7. Contour diagram of the residual in aspherical component of squared sound speed after subtracting the temporal average, as a function of radial distance and latitude using the MDI data. The solid lines show positive contours and dotted lines show negative contours. Contours are drawn at interval of $2 \times 10^{-5}$. The left panel shows the results using all modes with $1.5 \leq v \leq 3.5 \mathrm{mHz}$, while the right panel shows the results using modes with $1.5 \leq v \leq 3.0$ and $\ell<110$.

Waldmeier 1981; Makarov \& Sivaraman 1989a,b) and led to a picture where this latitude had surface flows diverging from it. In that case, the cells could act to dredge dynamo-generated flux from the lower convection zone into this latitude. On the other hand, ring diagram analyses of local helioseismic data (Basu et al. 1999; Haber et al. 2000) find poleward meridional circulations at low latitudes, in the outer $20 \mathrm{Mm}$ or so that they can image. Small-scale fields could be generated by the alpha effect operating in the upper convection zone, or could come from the decay of old sunspots and active regions. The meridional flows could sweep up this near-surface flux and collect it like jetsam in a downflow lane at $60^{\circ}$ latitude.

A further point of interest about $60^{\circ}$ latitude is that this is where the near-surface shear changes sign, according to MDI helioseismic measurements: the angular velocity increases with depth beneath the surface at low latitudes whereas it decreases with depth at high latitudes (Schou et al. 1998; Corbard \& Thompson 2002). This could reflect the role of differently signed meridional circulation flows in the transport of angular momentum on either side of $60^{\circ}$. Furthermore, the timedependence of rotation at high latitudes is perhaps another manifestation of the interplay between magnetic field and flows. The variation in rotation rate in the upper convection zone shows a pattern with bands of faster and slower than average rotation rate moving towards the equator with time at low latitudes (Howe et al. 2000; Antia \& Basu 2000) while at high latitudes these bands appear to be moving towards the pole (Antia \& Basu 2001). The transition between equatorward and poleward movement occurs around a latitude of $50^{\circ}$. The nearsurface rotation at high latitudes, as revealed by helioseismology, has been slowing down until 1999 during the rising phase of the present cycle (Schou 1999; Antia \& Basu 2001). At high latitudes, the rotation rate decreases with time, reaches a minimum and then starts increasing again. The epoch at which the minimum occurs is a function of latitude: it gradually shifts to later times as one goes to higher latitudes. One might envisage that it is the open magnetic field lines that slow the rotation. As the open flux retreats glacier-like back to high latitudes, it releases its brake on the rotation at a given latitude and so at that latitude the rotation rate springs back up.

One of the outstanding problems in solar physics is to identify the location of a plausible mechanism responsible for simultaneous variations of the Sun's luminosity, radius, effective temperature and $p$-mode frequencies with the activity cycle. The seat of the solar dynamo is generally believed to be located in the tachocline region beneath the convection zone where the mechanism for solar luminosity variation could also reside. However, it has been argued by Balmforth et al. (1996) that an agent like a magnetic field concentrated at the base of the solar convection zone is unlikely to be directly responsible for the observed cyclic modulations; nor can the purely thermal changes occurring in the near-surface layers of the Sun account for the observed irradiance variations. Any deeply seated driving mechanism would yield values of the quantity $W=(\mathrm{d} R / R) /(\mathrm{d} L / L)$, which measures the relative changes in solar radius and luminosity, somewhere between 0.2 and 0.5 . The current estimates of $W$ seem to suggest much lower values, of order 0.02 (Emilio et al. 2000). In fact, these results from satellite observations indicate that the principal cause of the solar luminosity variation is unlikely to be located very deep inside the Sun.

Helioseismic inversions have revealed the existence of a shear layer below the photosphere, extending to depths of about $50 \mathrm{Mm}$ below the photosphere, with its peak occurring at $30 \mathrm{Mm}$ depth, in the midst of the helium ionisation zone. (The above values depend somewhat on latitude, the depth of shear 
GONG $\delta c^{2} / c^{2}($ pre 1998.4)

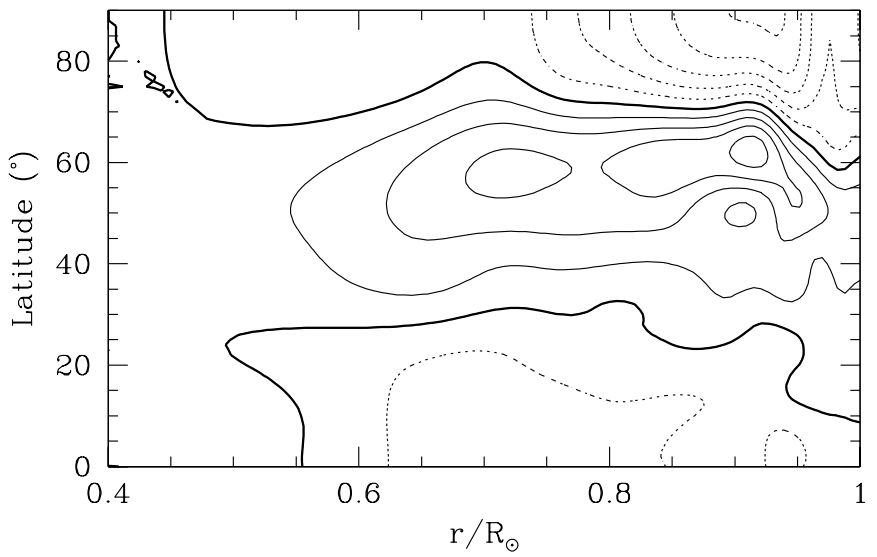

GONG $\delta c^{2} / c^{2}$ (post 1999.4)

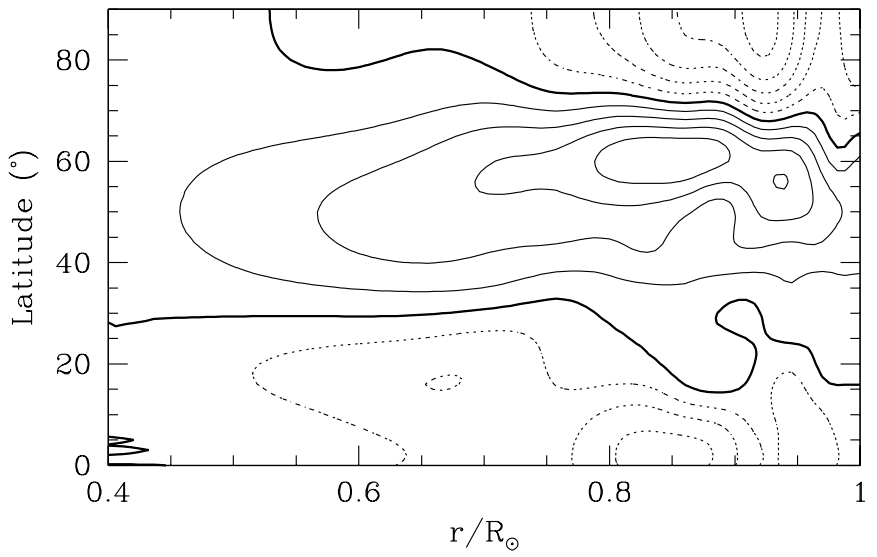

GONG difference

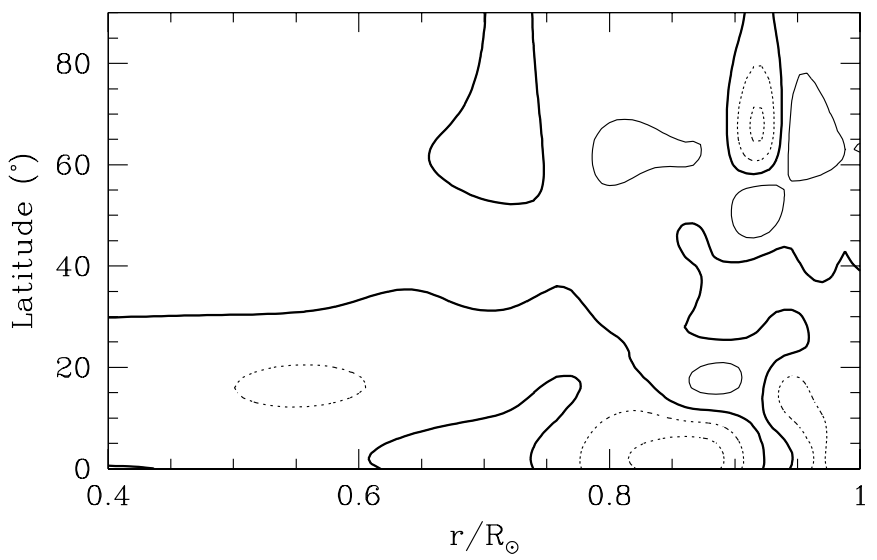

MDI $\delta c^{2} / c^{2}($ pre 1998.4)

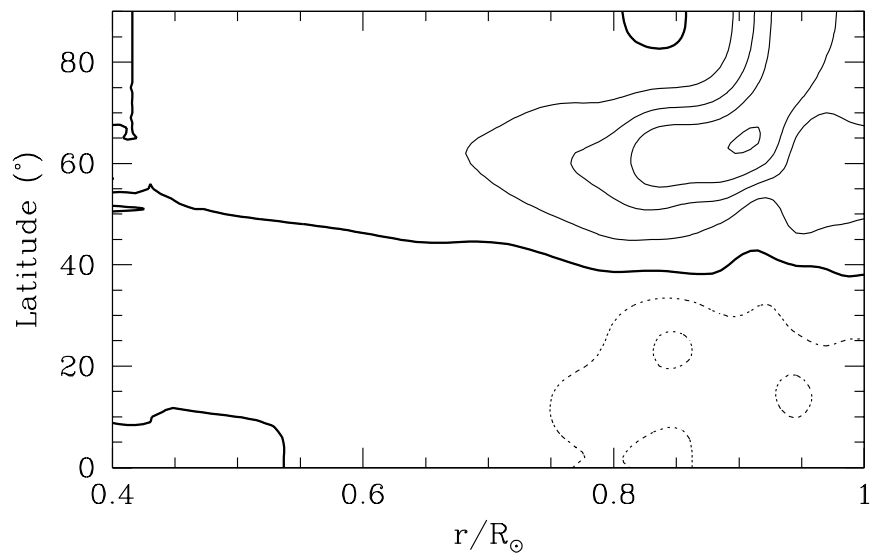

MDI $\delta c^{2} / c^{2}($ post 1999.4)

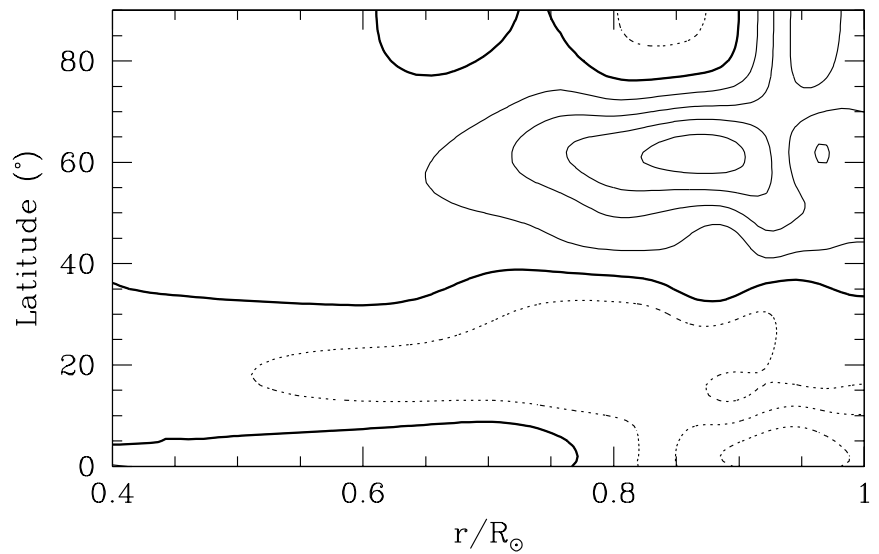

MDI difference

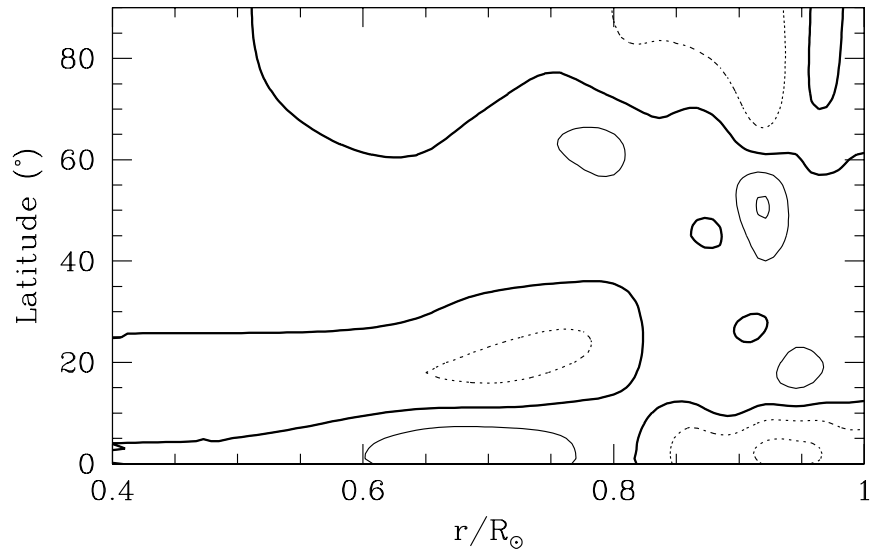

Fig. 8. Contour diagram of the temporal average of aspherical component of squared sound speed as a function of radial distance and latitude. The solid lines show positive contours and dotted lines show negative contours. Contours are drawn at interval of $2 \times 10^{-5}$. The thick contour in these figures mark the zero level. The temporal average using GONG data before 1998.4 (top-left), after 1999.4 (middle-left), and MDI data before 1998.4 (top-right), after 1999.4 (middle-right) are shown. The two panels at the bottom show differences in acoustic asphericity between the high and low activity averages for GONG (bottom-left) and MDI (bottom-right).

layer reducing with increasing latitude.) Dikpati et al. (2002) have explored the role of this subphotospheric radial shear layer in driving a solar dynamo, and find that a differentially rotating shear layer is capable of generating toroidal magnetic fields of up to $1 \mathrm{kG}$ strength. Antia et al. (2000) had invoked the pumping of downward moving plumes (downdrafts) to concentrate toroidal magnetic field in the outer convection zone and to enhance its strength to values approaching $70 \mathrm{kG}$ at depths around $70 \mathrm{Mm}$ below the surface. This takes place at depths around $70 \mathrm{Mm}$ below the surface, where some sort of equipartition is achieved between the magnetic energy density $\left\langle B^{2} / 8 \pi\right\rangle$ and the kinetic energy density of downdrafts $\left\langle\rho w^{2}\right\rangle$. 
Note, the velocities of the order of $1 \mathrm{~km} \mathrm{~s}^{-1}$ adopted here, refer to the downdraft motions which have velocities several times those given by the standard mixing length theory for convective elements (cf., Mestel 1999). This process, of course, does not exclude the generation of a large fraction of the toroidal magnetic field in the solar interior, with the help of differential rotation in the tachocline. We are merely pointing out that part of the toroidal field in the convection zone may be generated in the sub-surface shear layer itself, where the poloidal field could be reinforced by some kind of alpha effect. This would then be immediately available for further generation of the toroidal field by the action of strong subsurface radial shear.

Earlier it was noted by Antia et al. (2000) and Dziembowski et al. (2000) that residual splitting coefficient $a_{2}$ showed a striking peak around $r=0.96 R_{\odot}$. If this peak should result solely from the presence of large-scale magnetic field at depths of order $30 \mathrm{Mm}$, the inferred magnetic field strength comes out to be $20-30 \mathrm{kG}$. But with the removal of surface term, this peak shifts downwards to $70 \mathrm{Mm}$ depth and required magnetic field strength would be about $70 \mathrm{kG}$.

It is readily seen from Fig. 1 that the contours of constant $\delta c^{2} / c^{2}$ in both the GONG and MDI data show a pronounced radial extension around the $60^{\circ}$ latitude peak situated at a depth of about $70 \mathrm{Mm}$. Even though a comparison of the contours of constant $\delta c^{2} / c^{2}$ in the pre-1998.4 and post 1999.4 GONG and MDI data sets displayed in Fig. 8 barely shows a detectable temporal variation, the difference in acoustic asphericity between the high and low activity averages shown in the bottom panels of Fig. 8 has some indication of a possible temporal variation of asphericity around a depth of $0.1 R_{\odot}$ at both equatorial and higher latitudes. It may therefore be tempting to speculate that there is a reservoir of magnetic energy stored in the outer $0.1 R_{\odot}$ of the convection zone which perhaps undergoes some temporal variation. Such a time-varying storage of magnetic energy might even play a role in driving the solar activity cycle. We suggest that the solar irradiance variation is probably due to the periodic release of such a stored magnetic energy in the outer layers of the Sun, resulting from an interplay between the solar magnetic field, subsurface radial shear and pumping by convective downdrafts.

Acknowledgements. This work utilises data obtained by the Global Oscillation Network Group (GONG) project, managed by the National Solar Observatory, which is operated by AURA, Inc. under a cooperative agreement with the National Science Foundation. The data were acquired by instruments operated by the Big Bear Solar Observatory, High Altitude Observatory, Learmonth Solar Observatory, Udaipur Solar Observatory, Instituto de Astrofisico de Canarias, and Cerro Tololo Inter-American Observatory. This work also utilises data from the Solar Oscillations Investigation/ Michelson Doppler Imager (SOI/MDI) on the Solar and Heliospheric Observatory (SOHO).
SOHO is a project of international cooperation between ESA and NASA. SMC is grateful to DAE-BRNS for support under the Senior Scientist Scheme and to Ian Roxburgh for supporting his visit to Queen Mary, University of London under the Leverhulme Trust Visiting Professorship scheme.

\section{References}

Antia, H. M. 2002, in Proc. IAU Colloq., 188, Magnetic Coupling of the Solar Atmosphere, ESA SP-505 [astro-ph/0208339]

Antia, H. M., \& Basu, S. 2000, ApJ, 541, 442

Antia, H. M., \& Basu, S. 2001, ApJ, 559, L67

Antia, H. M., Chitre, S. M., \& Thompson, M. J. 2000, A\&A, 360, 335

Antia, H. M., Basu, S., Hill, F., et al. 2001, MNRAS, 327, 1029

Balmforth, N. J., Gough, D. O., Merryfield, W. J. 1996, MNRAS, 278, 437

Basu, S., Antia, H. M., \& Tripathy, S. C. 1999, ApJ, 512, 458

Christensen-Dalsgaard, J., Gough, D. O., \& Thompson, M. J. 1991, ApJ, 378, 413

Corbard, T., \& Thompson, M. J. 2002, Sol. Phys., 205, 211

Dikpati, M., Corbard, T., Thompson, M. J., \& Gilman, P. A. 2002, ApJ, 575, L41

Dziembowski, W. A., Goode, P. R., Kosovichev, A. G., \& Schou, J. 2000, ApJ, 537, 1026

Emilio, M., Kuhn, J. R., Bush, R. I., \& Scherrer, P. 2000, ApJ, 543, 1007

Gough, D. O. 1977, in The energy balance and hydrodynamics of the solar chromosphere and corona, ed. R. M. Bonnet \& P. Delache (G. de Bussac, Clermont-Ferrand), Proc. IAU Colloq., 36, 3

Gough, D. O. 1981, in Variations of the Solar Constant, ed. S. Sofia (NASA CP 2191) 185

Gough, D. O., Kosovichev, A. G., Toomre, J., et al. 1996, Science, 272, 1296

Haber, D. A., Hindmann, B. W., Toomre, J., Bogart, R. S., \& Hill, F. 2001, in Proc. SOHO 10 / GONG 2000 Workshop, ed A. Wilson (ESA SP-464) 213

Harvey, J. W., Hill, F., Hubbard, R., et al. 1996, Science, 272, 1284

Hill, F., Stark, P. B., Stebbins, R. T., et al. 1996, Science, 272, 1292

Howe, R., Christensen-Dalsgaard, J., Hill, F., et al. 2000a, ApJ, 533, L163

Leroy, J.-L., \& Noens, J.-C. 1983, A\&A, 120, L1

Li, L. H., \& Sofia, S. 2001, ApJ, 549, 1204

Makarov, V. I., \& Sivaraman, K. R. 1989a, Sol. Phys., 119, 35

Makarov, V. I., \& Sivaraman, K. R. 1989b, Sol. Phys., 123, 367

Mestel, L. 1999, Stellar Magnetism (Clarendon press Oxford) 306

Scherrer, P. H., Bogart, R. S., Bush, R. I., et al. 1995, Sol. Phys., 162, 129

Schou, J. 1999, ApJ, 523, L181

Schou, J., Christensen-Dalsgaard, J., \& Thompson, M. J. 1994, ApJ, 433, 389

Schou, J., Antia, H. M., Basu, S., et al. 1998, ApJ, 505, 390

Ulrich, R. K., \& Rhodes, E. J. 1977, ApJ, 218, 521

Waldmeier, M. 1981, Sol. Phys., 70, 251 